\title{
A Solution for Water Factories in Vietnam using Automatic Meter Reading Technology
}

\author{
Vu Chien Thang \\ Faculty of Electronics and Communications Technology, University of Information and Communications Technology, \\ Thai Nguyen, 250000, Viet Nam \\ E-mail: vcthang@ictu.edu.vn
}

Received: 21 March 2018; Accepted: 22 June 2018; Published: 08 August 2018

\begin{abstract}
A solution for water factories in Vietnam using automatic meter reading technology is proposed in this paper. The water meters and water quality meters for water factories in Vietnam have been designed and prototyped. Water meters differ from conventional meters in that they record the water consumption and communicate that information back to the water suppliers for monitoring and billing without the need for manual readings. Water quality meters monitor multiple parameters relating to water quality such as $\mathrm{pH}$, electroconductivity, temperature, etc., and transmit these parameters to a server via GPRS network. Therefore, customers and water suppliers can monitor the water consumption, water quality parameters from anywhere via the Internet. This project has been conducted in a water factory located in Thai Nguyen Province, Vietnam. The results have shown that the consideration of this solution has been operated well in the real condition in Vietnam.
\end{abstract}

Index Terms-Smart water meter, water quality meter, smart water sensor, the solution for water factory.

\section{INTRODUCTION}

Currently, manual water meter reading is still widely used in Vietnam. This manual method is waste of time and human labor. Automatic Meter Reading (AMR) is the technology that automatically collects consumption, diagnostics, and status of the water meter and transmits the data to a central database for billing, troubleshooting, and analyzing. This method supports vendors to save the expense of periodic trips to each physical location to read a meter. Another advantage of this technology is that billing can be based on real-time consumption. This paper presents the solution for water factories in Vietnam in two following parts:

In the first part, a system model of water quality monitoring is proposed. This system automatically collects physical and chemical parameters in the tanks at the water factory. Figure 1 shows the model of water quality monitoring. Every a few hours, the smart water sensors measure different water quality parameters in the tanks. Data is measured, digitized, and transmitted to the server via GPRS network by smart water sensors.
The second part of study recommends a system model of smart water metering. This system automatically collects water consumption and transmits the data to a central database for billing. Figure 2 shows the model of smart water metering system. The smart water meters periodically measure water consumption and send the data to an internet gateway via ZigBee/IEEE802.15.4 network. The internet gateway is used in this project to gather all the data from the smart water meters and transmits them to the server at the water factory.

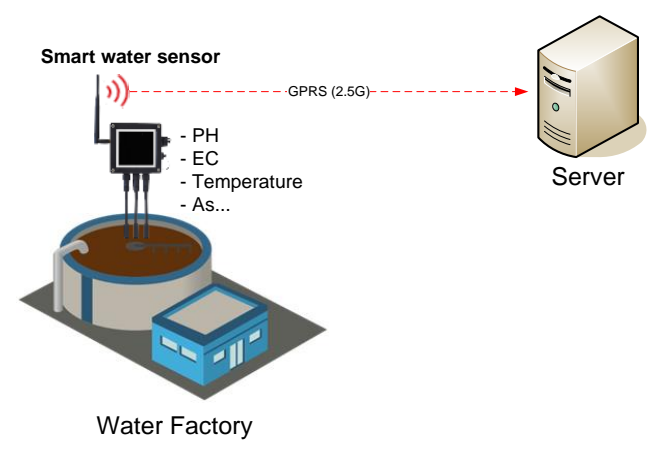

Fig.1. Model of Water Quality Monitoring

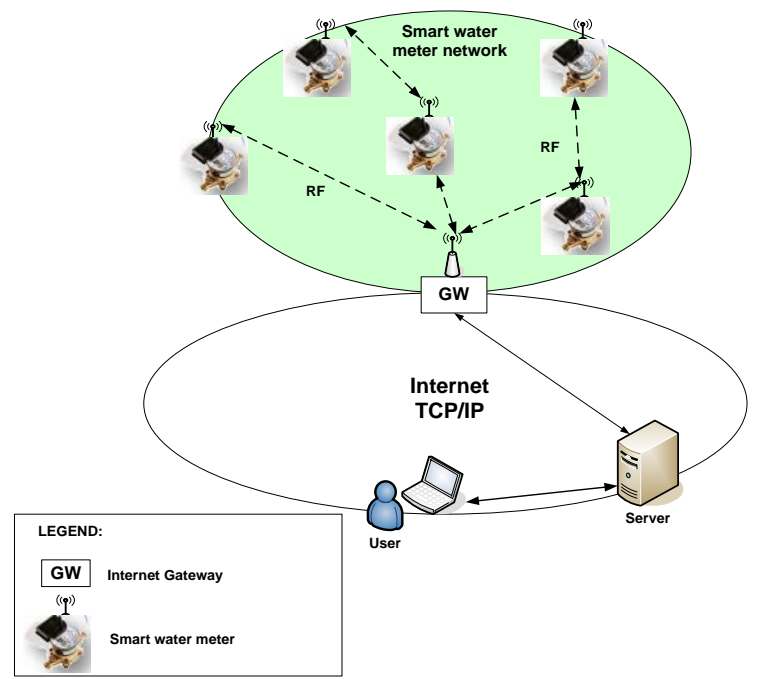

Fig.2. Model of Smart Water Metering. An Internet Gateway Is Deployed To Collect Data from Customers

The remainder of this paper is organized as follows. Section II shows the related works. Section III introduces 
the design solution of the smart water sensor. Design solutions of smart water metering system are presented in section IV, and section V. Section VI presents the data management software. Finally, Section VII outlines the main conclusions and contribution of the study.

\section{RELATED WORKS}

Advanced Metering Infrastructures (AMI) are comprised of hardware and software that create a network between advanced meters at the customer site and a service provider, such as an electric, gas, or water utility. These meters have the ability to transmit the collected data through commonly available networks such as power line communications, WiFi, ZigBee, GSM/GPRS. The meter data is received by the smart meters and sent to the Meter Data Management System (MDMS) that manages data storage and analyses data to provide the information in a useful form to users [1]. In [2], authors proposed an integrated Internet of Things (IoT) architecture for electricity, water, and gas smart metering, which benefits both the customers and the utilities.

The term Automatic Meter Reading (AMR) came about in the middle 1980s, and more prominently in the early 1990s. The goal of AMR system is to help collect the meter measurement automatically and possibly send commands to the meters. All AMI systems contain AMR functionality.

In $[3,4]$ authors provided extensive coverage of the AMR/AMI system, starting from discussing the potential benefits and past development stages to giving directions of future generations of AMR/AMI. They also presented four major types of AMR communication networks: power line carrier (PLC), the cellular network, telephone/internet, and short-range radio frequency such as WiFi, Bluetooth, ZigBee. At present, the wireless technology is widely used in ways, compared with the wired devices the wireless ones are low cost and easy to carry. Besides, the structure of a wireless AMR system is also simple and can save much labor force and resources. In [5], authors introduced a system, made to express the success and efficiency of automatic meter reading, through GSM wireless technology. In [6], authors proposed system automatically reads the consumption energy and sends it to the electric provider by using short messaging service. In [7], an automatic meter reading system focus on the design of an energy meter executed with ZigBee wireless communication. In [8], authors proposed a centralized automatic meter reading system based on the application of GPRS wireless communication technology. This system enables to reduce the electric power enterprise human resources cost, improve work efficiency and quality of reading business, create the more economic and social benefits for the enterprise, to promote the sustainable development of power industry.

In [9], a prototype demonstrator of a smart water metering solution, developed in cooperation with Telecom Italia Lab, and built upon self-powered nodes in a WM-Bus capillary network at $169 \mathrm{MHz}$ has been presented. The smart water meter collects water consumption through a hall-effect sensor and data are transmitted to an aggregator as a gateway to the GSM/GPRS network. In [10], authors designed a smart sensor network that can conduct online measurements of the water quality parameters according to the rules and standards.

In [11], authors proposed other system based on hybrid systems that can make use of robust networking topologies such as the GSM and ZigBee. In this proposed system, ZigBee module will be attached to the meter by using interface board and the data collector will be connected to the central computer by using GSM. With this system, the power company can save cost in doing meter reading and provide better services to their customers.

Broadband over Power-Line (BPL) $[12,13]$ is another typical medium for data communication over power lines. Bi-directional broadband communication can be achieved by merging BPL technology and smart water meter systems. The BPL-based system has following advantage: no new wire, covering a wide range of convenient connections, the reliability of access and high speed. However, a BPL-based system is an expensive system because it requires other components such as the collector, concentrator and master station.

\section{SMART WATER SENSOR}

\section{A. Description}

In this project, the smart water sensor has some functions:

- Measuring different water quality parameters automatically in the tanks such as temperature, electro-conductivity, $\mathrm{pH}$, etc. (potentially extended to other types of sensors via expansion ports).

- Sending data automatically to the web server via GPRS network.

\section{B. Hardware Solution}

Figure 3 shows the block diagram of the smart water sensor.

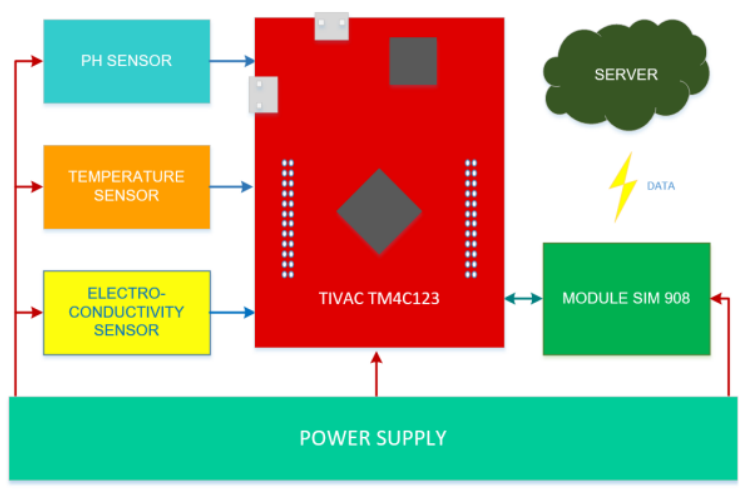

Fig.3. The Functional Block Diagram of the Smart Water Sensor 
- Power Supply: Using LM78M05 and LM2596 that are produced by Texas Instruments. LM78M05 provides an output voltage of $5 \mathrm{~V}$ for KIT TivaC TM4C123GXL [14] and sensors. LM2596 provides an output voltage of $4.2 \mathrm{~V}$ and an output current of 3A for Sim 908 module.

- Sensors Block: This block includes three sensors which are $\mathrm{PH}$, electroconductivity, and temperature sensor. This block is connected to TivaC TM4C123GXL via jumpers.

- TivaC Microcontroller Kit: A product of Texas Instrument namely TivaC TM4C123GXL is used. This is an ultra-low-power microcontroller. TivaC TM4C123GXL receives data from sensors, configures the SIM module, and sends data to the web server via GPRS network.

Figure 4 shows the printed circuit for the smart water sensor.
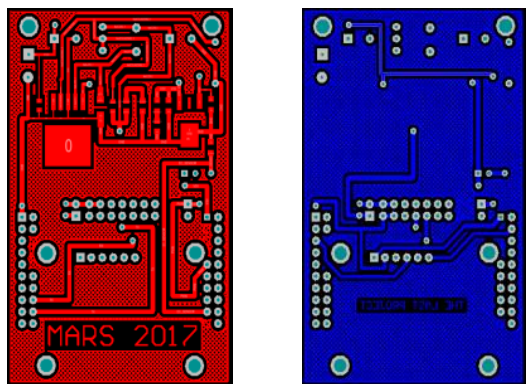

Fig.4. Printed Circuit for the Smart Water Sensor (Top and Bottom Layer)

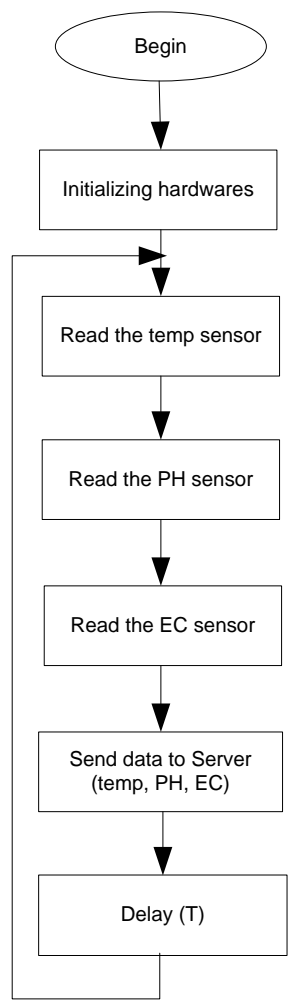

Fig.5. Embedded Software Algorithm for the Smart Water Sensor

\section{Embedded Software Solution for Smart Water Sensor}

The smart water sensor periodically measures different water quality parameters (such as $\mathrm{pH}$, temperature, EC) in the tank. These parameters are measured, digitized, and transmitted to the server via GPRS network. Figure 5 illustrates the embedded software algorithm for the smart water sensor.

\section{Experiment Results}

Figure 6 illustrates the smart water sensor. The smart water sensor was deployed in the real condition as shown in figure 7 .

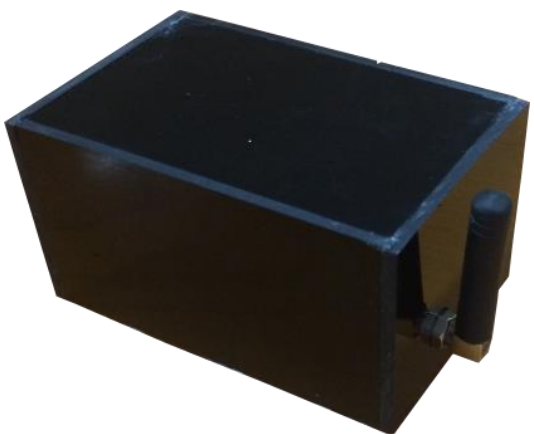

Fig.6. Smart Water Sensor

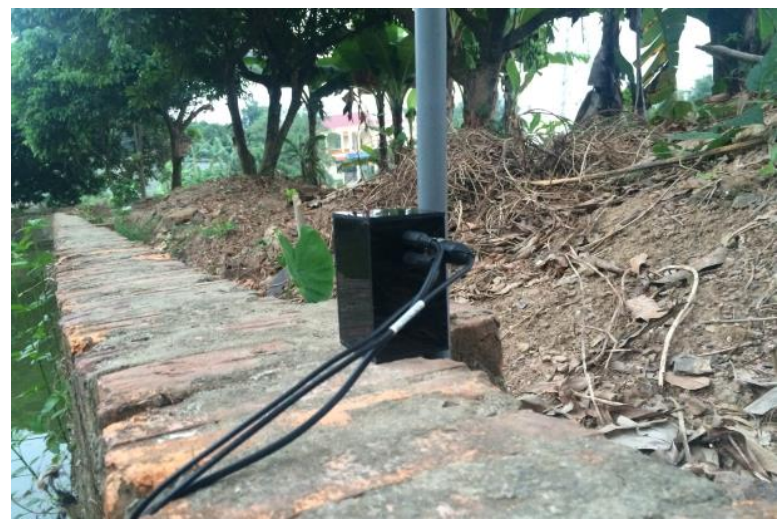

Fig.7. Deploying the Smart Water Sensor in a Real Condition

The water quality parameters are sent to the server. Water suppliers can monitor these parameters from anywhere via the Internet as shown in figure 8 .

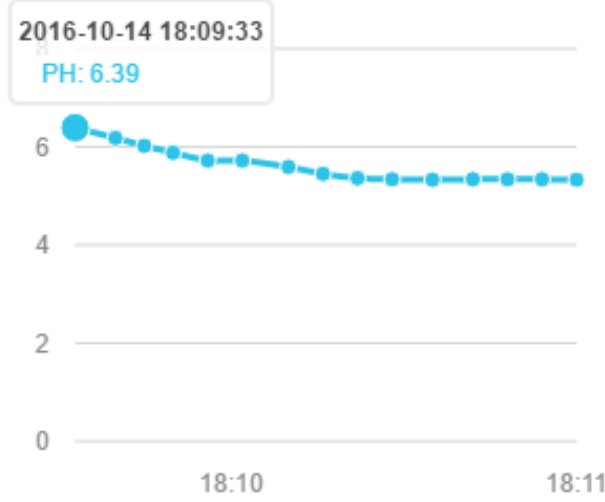

Fig.8. Chart of the Water Quality Parameter 


\section{SMART WATER METER}

\section{A. Description}

The smart water meter has several functions which are:

- Measuring the water flow automatically.

- Sending the water consumption periodically to the server via Zigbee networks.

\section{B. Hardware Solution}

Figure 9 shows the block diagram of the smart water meter.

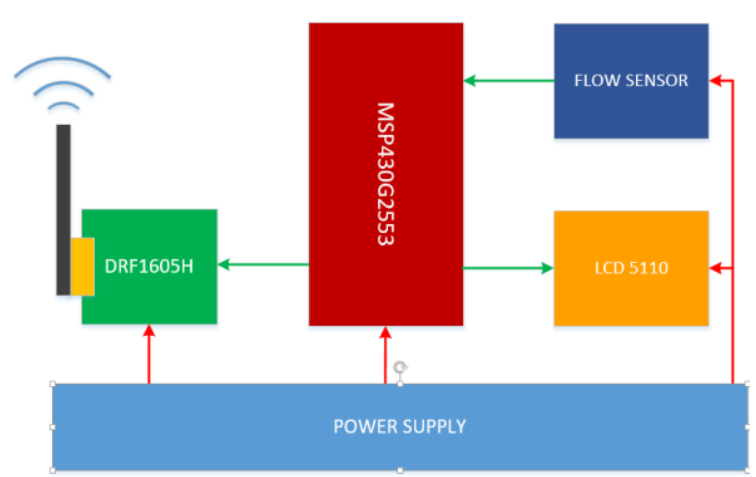

Fig.9. The Functional Block Diagram of the Smart Water Meter

- TI microcontroller MSP430G2553: A chip from Texas Instrument namely MSP430G2553 [15] is used. This is an ultra-low-power microcontroller, and suitable for the smart water meter.
MSP430G2553 receives the water flow data from the flow sensor, sends the data to ZigBee network and controls LCD screen.

- Water Flow Sensor: Using YF - S201 sensor [16] to measure the water flow. It is connected to MSP430G5223.

- LCD Nokia 5110: The water flow is displayed on LCD Nokia 5110 [17].

- Power Supply: Using LM1117 and LM7805 produced by TI. LM1117 provides an output voltage of $3.3 \mathrm{~V}$ for the microcontroller, LCD, and DRF1605H. LM7805 provides an output voltage of $5 \mathrm{~V}$ for the water flow sensor.

- DRF1605H Module: This is a communication module using CC2530 [18] chip from Texas Instruments with IEEE 802.15.4 communication standards, characteristics of ZigBee technology is a low transmission speed, low energy consumption, and low cost. Therefore, it is suitable for the smart water meter.

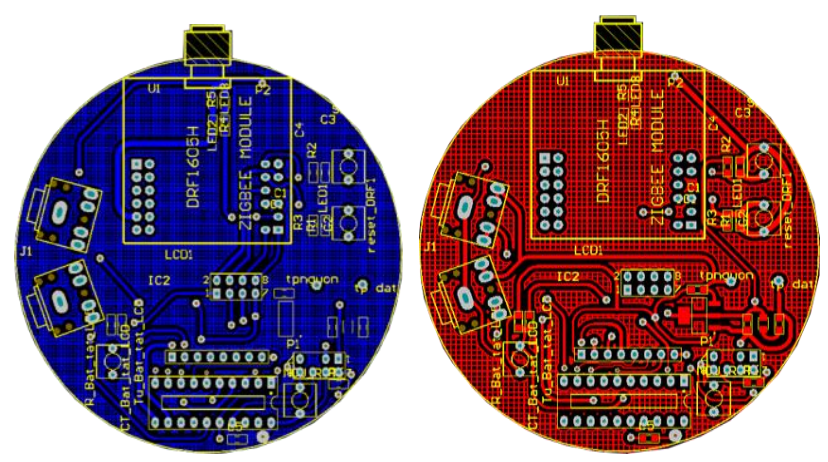

Fig.10. Printed Circuit for the Smart Water Meter (Top and Bottom Layer)

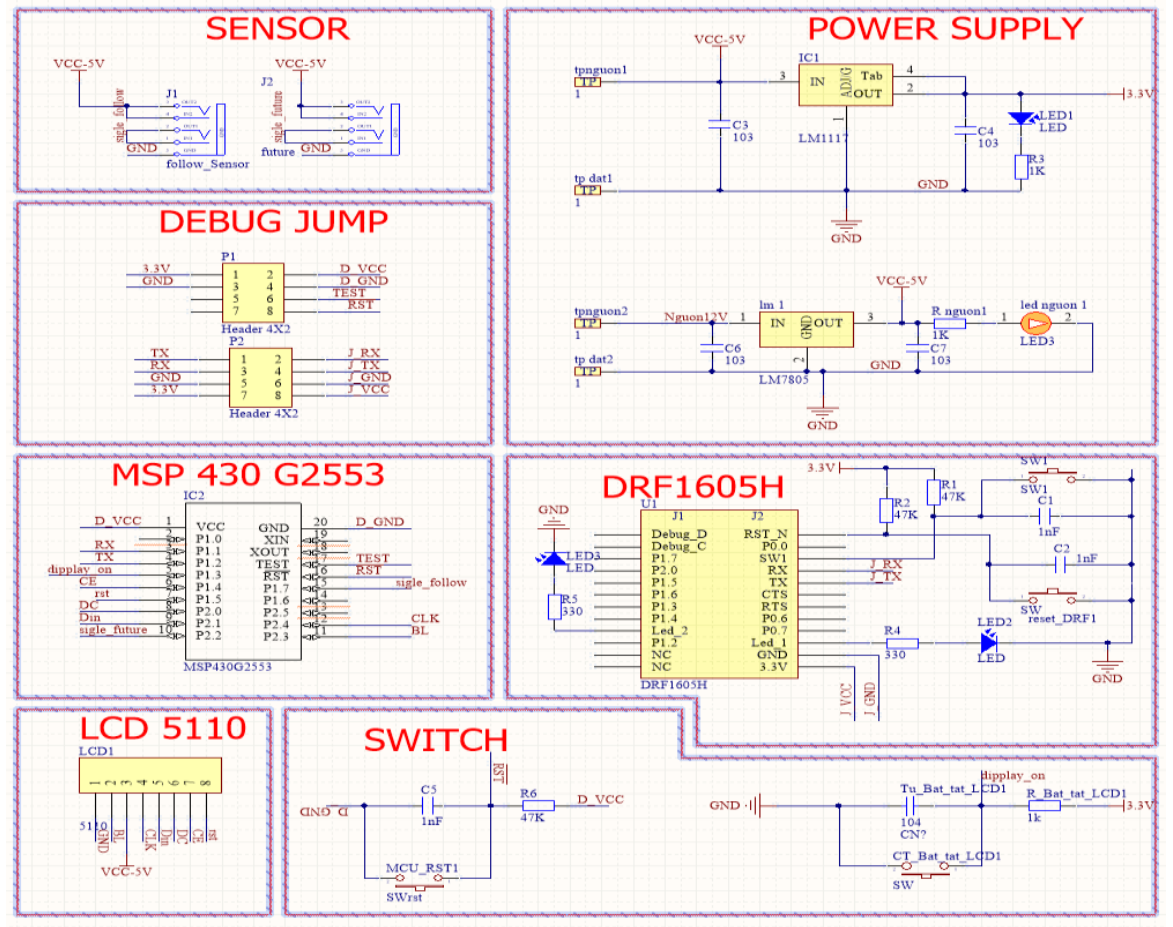

Fig.11. Schematic for the Smart Water Meter 
Figure 10, 11 show the printed circuit and schematic for the smart water meter.

\section{Embedded Software Solution for Smart Water Meter}

Figure 12 illustrates the embedded software algorithm for the smart water meter.

Figure 13 illustrates the algorithm of timer interrupt service process for the smart water meter. Smart water meter periodically sends data to the internet gateway.

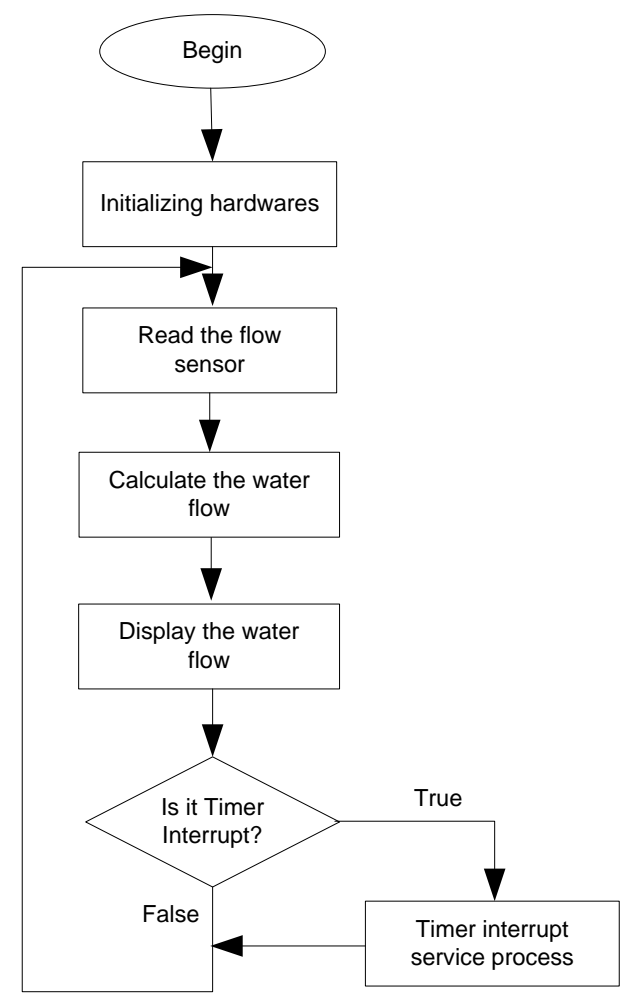

Fig.12. Embedded Software Algorithm for the Smart Water Meter

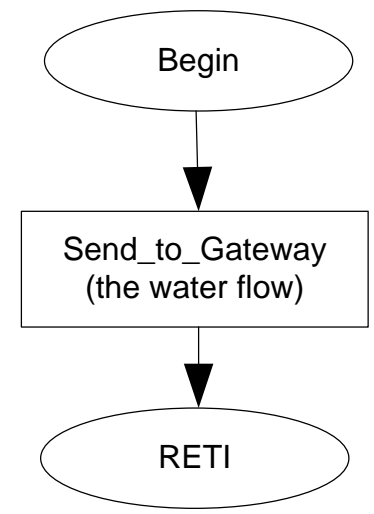

Fig.13. The Algorithm of Interrupt Service Routine for the Smart Water Meter

\section{Experiment Results}

Figure 14 illustrates the smart water meter.

The smart water meter was deployed in the real condition as shown in figure 15. Customers can monitor water consumption on the LCD screen of this device.

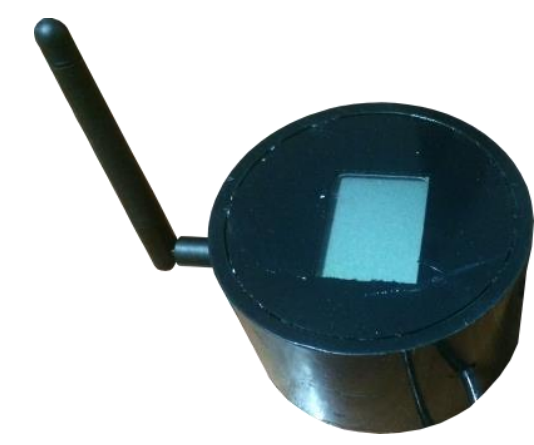

Fig.14. Smart Water Meter

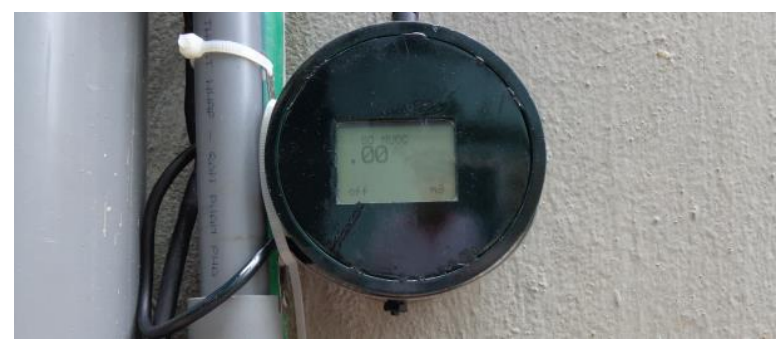

Fig.15. Deploying the Smart Water Meter in a Real Condition

\section{INTERNET GATEWAY}

\section{A. Description}

The gateway collects the data automatically from the smart water meters and sends the data to the web server via an internet connection. Therefore, the gateway must support two communication protocols which are Ethernet and ZigBee.

\section{B. Hardware Solution}

Figure 16 shows the block diagram of internet gateway. The detail descriptions of blocks in figure 16 include:

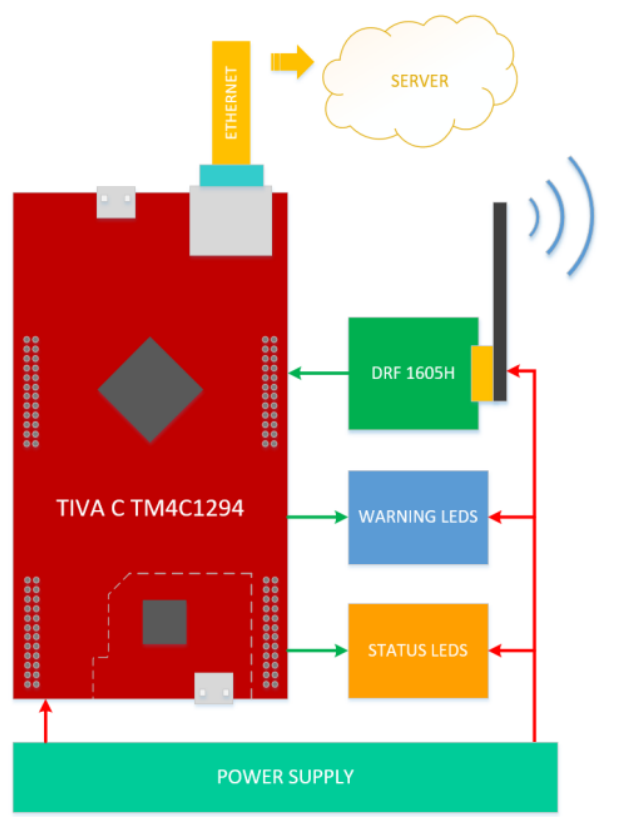

Fig.16. The Functional Block Diagram of the Internet Gateway 
- TM4C1294 KIT: This kit is produced by Texas Instruments. The TM4C1294 Kit integrates a variety of peripherals enabling internet of things gateway applications [19]. There are a lot of communication interfaces on board such as 10/100 Ethernet MAC and PHY, USB 2.0, and a multitude of simultaneous serial connectivity. TM4C1294 has low cost, low power consumption, large memory, which combines with ethernet interface on board. Therefore, TM4C1294 is chosen in the internet gateway design.

- Power Supply: Using LM1117 and LM7805 produced by Texas Instruments.

- DRF1605H Module: It is configured as a coordinator in the ZigBee network. This module is connected to the microcontroller via UART ports.

\section{Embedded Software Solution for Internet Gateway}

Figure 17 illustrates the embedded software algorithm for the internet gateway. The internet gateway is shown in figure 18 .

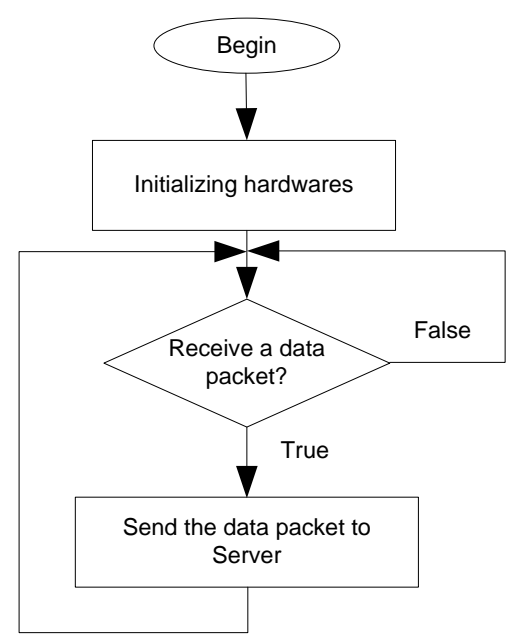

Fig.17. Embedded Software Algorithm for the Internet Gateway

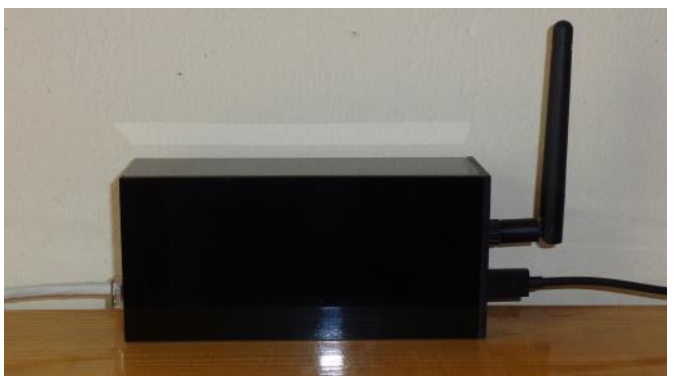

Fig.18. Internet Gateway

\section{WEB APPLICATION}

A website deployed on the server has been designed. Data is stored in the database and updated on the website. Customers and water suppliers can monitor water consumption, water quality parameters everywhere via the Internet.
Water suppliers can:

- Manage the smart water meters and customers.

- Monitor the total water consumption of factory.

- Monitor the water quality.

- Look up the water bill of a customer.

- Update the information of a customer.

Customers can:

- Monitor the water quality.

- Look up the water bill.

Customers and water suppliers can log in the system as shown in figure 19. Figure 20 illustrates the water bill of a customer.

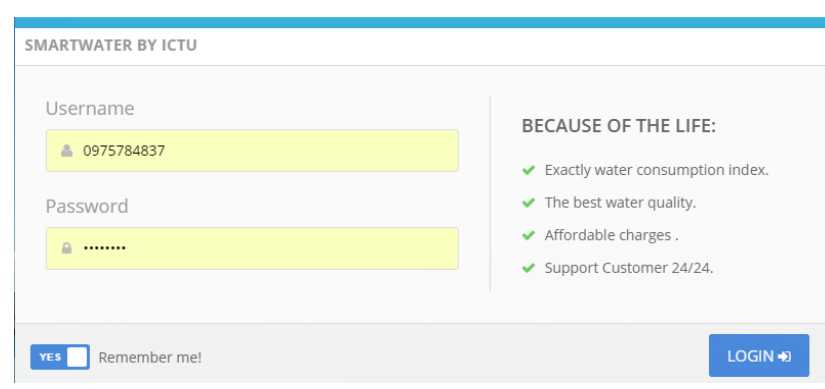

Fig.19. Login the System

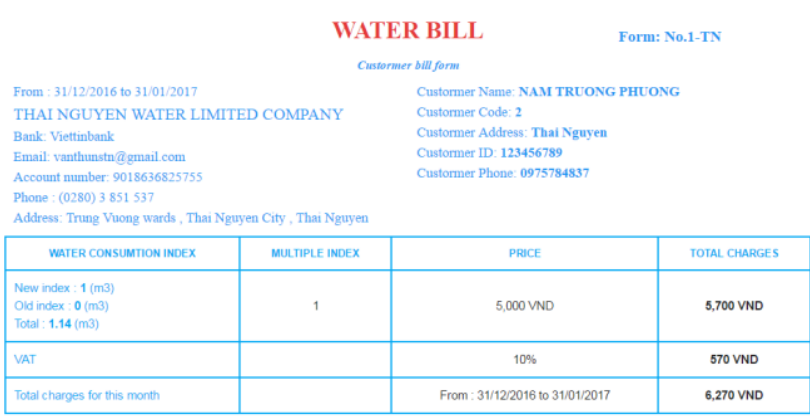

Fig.20. The Water Bill of a Customer

Figure 21 illustrates the water quality parameters. Customers and water suppliers can monitor these parameters from anywhere via the Internet.

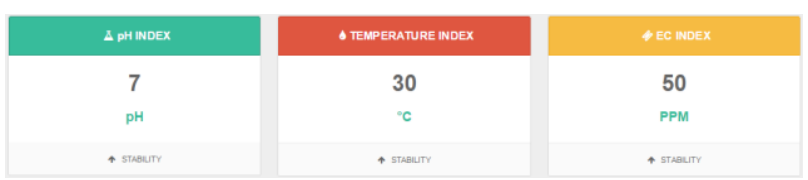

Fig.21. The Water Quality Parameters

\section{CONCLUSION}

Automatic Meter Reading Technology supports water vendors in Vietnam to save the expense of periodic trips to each physical location to read a meter. In this paper, water meters, and water quality meters for smart water application have been designed and prototyped. A website was developed to collect water consumption, and 
water quality parameters. This solution allows users to monitor water consumption and water quality parameters from any places via the Internet. This study has provided some insights into the potential solution applying for the water factories in Vietnam. It would seem that this technology can be systematically and successfully applied to real conditions.

\section{ACKNOWLEDGMENT}

I would like to thank all members of the Faculty of Electronics and Communication Technology for their technical support. I also would like to express my highest regards and thanks to Thainguyen University for funding my work under the project titled Research and Development of an Intelligent System for Automatic Metering in Water Factories (ĐH2017-TN07-01.ĐH).

\section{REFERENCES}

[1] Omowunmi M. Longe, Khmaies Ouahada, Hendrick C. Ferreira, Suvendi Rimer, "Wireless Sensor Networks and Advanced Metering Infrastructure Deployment in Smart Grid," Proceedings of International Conference on eInfrastructure and e-Services for Developing Countries, pp. 167-171, 2013.

[2] Jaime lioret, Jesus Tomas, Alejandro Canovas, and Lorena Parra, "An integrated IoT Architecture for Smart Metering," IEEE Communications Magazine, volume 54, issues 12, pp. 50-57, 2016.

[3] Tarek Khalifa, Kshirasagar Naik, Amiya Nayak, "A Survey of Communication Protocols for Automatic Meter Reading Applications," in IEEE Communications Surveys \& Tutorials, vol.13, no.2, pp.168-182, 2011.

[4] Ramyar R Mohassel, ALan Fung, Farah Mohammadi, Kaamran Raahemifar, "A Survey on Advanced Metering Infrastructure," Electrical Power and Energy System Journal, pp.473-484, 2014.

[5] H.G. Rodney Tan, C.H. Lee and V.H. mork, "Automatic power meter reading systems using GSM network," 8th International Power Engineering Conference, pp.465-469, 2007.

[6] Ashna K, Sudhish N. Gorgre, "GSM based automatic energy meter reading system with instant billing," IEEE International conference held at Kottayam, pp. 65-72, 2013.

[7] Champ Prapasawad, Kittitachpornprasitpol, Wanchalermpora, "Development of an Automatic meter reading system based on ZigBee pro smart energy profile IEEE 802.15.4 standard," International Conference on Electronic Devices and Solid State Circuit (EDSSC), pp.13, Dec 2012.

[8] LiuNan, WangYang, LiuShanShan, "Centralized automatic meter reading system based on GPRS technology," International Conference on Instrumentation \& Measurement, Computer, Communication and Control, pp. 549-553, 2016.

[9] Gabrielli, Pizzichini, Spinsante, Squartini, Gavazzi,
"Smart water grids for smart cities: A sustainable prototype demonstrator," Proceedings of 2014 European Conference on Networks and Communications, pp.1-5, 2014.

[10] Cloete Niel Andre, Malekian Reza, and Nair Lakshmi, "Design of smart sensors for Real-Time water Quality Monitoring," IEEE Access, volume 4, 2016.

[11] Aryo Handoko Primicanta, Mohd Yunus, and Mohammad Awan, "Hybrid Automatic Meter Reading System," Proceedings of the IEEE International Conference on Computer Technology and Development, vol.2, pp. 264267, 2009.

[12] Qingyang Liu, Bingzhen Zhao, Yirong Wang, Jing Hu, "Experience of AMR systems based on BPL in China," Proceedings of the IEEE International Symposium on Power Line Communications and Its Applications, pp. 280-284, 2009.

[13] Mingfu Li and Hung-Ju Lin, "Design and Implementation of Smart Home Control Systems Based on Wireless Sensor Networks and Power Line Communications," IEEE Transactions on Industrial Electronics, pp. 44304442, 2014.

[14] User's guide, retrieved date: [15,June,2017], online available at: http://www.ti.com/ lit/ug/spmu296/spmu296.pdf

[15] User's guide, retrieved date: [15,June,2017], online available at: http://www.ti.com/lit/ds/ symlink/msp430g2553.pdf

[16] Retrieved date: [15,June,2017], online available at: http://www.amazon.in/Robodo-Electronics-YF-S201Water-Sensor/dp/B00REJ7QF2

[17] Retrieved date: [15,June,2017], online available at: http://www.amazon.in/REES52-REES52-6-NokiaModule-Backlight/dp/B00YSG0K98/ref=sr_1_1?s= industrial\&ie $=$ UTF8 \&qid $=1497519985 \& \mathrm{sr}=11 \&$ keyword $\mathrm{s}=\mathrm{LCD}+\mathrm{Nokia}+5110$

[18] User's guide, retrieved date: [15,June,2017], online available at: http://www.ti.com/lit/ds/ symlink/ cc2530.pdf

[19] User's guide, retrieved date: [15,June,2017], online available at: http://www.ti.com/lit/ug/ spmu365c/spmu365c.pdf

\section{Authors' Profile}

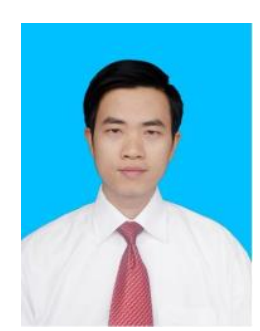

Vu Chien Thang received the MSc degree in Electronics and Communication Technology in 2009 from Hanoi University of Science and Technology and Ph.D. in Telecommunication Engineering in 2015 from Vietnam Research Institute of Electronics, Informatics, and Automation. He is currently a lecturer at Thainguyen University of Information and Communication Technology. His research interests include wireless sensor networks, internet of things, embedded systems.

How to cite this paper: Vu Chien Thang,"A Solution for Water Factories in Vietnam using Automatic Meter Reading Technology", International Journal of Computer Network and Information Security(IJCNIS), Vol.10, No.8, pp.44-50, 2018.DOI: $10.5815 / \mathrm{ijcnis.2018.08.05}$ 